\title{
Beispiel Wortbildung - Die Erhebung und Interpretation von Daten
}

\author{
HILKE ELSEN \& SASCHA MICHEL
}

\section{Problemstellung}

Die Wortbildung ist seit langem als etablierte linguistische (Teil-)Disziplin zu betrachten, die sich nicht nur auf die den Terminus umfassenden prozessualen und resultativen Ausprägungen aus synchroner Perspektive beschränkt, sondern auch umfangreiche diachrone sowie Fragen zur Lehn- und Fremdwortbildung in den Fokus rückt. Forschungsgeschichtlich relevant ist dabei die Konzentration auf sprachsystematische Problem- und Fragestellungen unter weitgehender Vernachlässigung des Sprachgebrauchs. Untersuchungen stellen primär die Dokumentation und Erklärung des Resultats und des Prozesses wortbildnerischer Tätigkeit in den Mittelpunkt, um den (festen) Sprachbestand eingehend $\mathrm{zu}$ erfassen. Dies bedeutet, dass zur Generierung möglichst homogener Klassen und zur Beschreibung von „,interferenzfreien“ Bildungsregeln von zugrunde liegenden Daten und Ergebnissen abstrahiert wird. Diese Vorgehensweise steht zunächst im Einklang mit de Saussures Langue ${ }^{1}$ Postulat (de Saussure 1969) und Chomskys Konzeption einer generativen Grammatik (Chomsky 1971), macht jedoch nicht deutlich, in welcher Weise Langue bzw. Kompetenz mit Parole bzw. Performanz interagieren. Es bleibt demnach offen, ob Sprachsystem und Sprachgebrauch zwei distinkte Erscheinungsformen darstellen, die unabhängig voneinander beschrieben werden müssen (wobei der Langue bzw. der Kompetenz eine stärkere Bedeutung zukommt), oder ob es unmöglich erscheint, „eine klare Grenze zwischen kontextunabhängiger Grammatik (Kompetenz) und kontextabhängiger Interpretation (Performanz) zu ziehen“ (Levinson 1994: 8). Die Frage, was zuerst da ist, Langue oder Parole, wurde ohnehin bisher nicht eindeutig beantwortet. Außerdem ist die Annahme einer - nie erwiesenen - angeborenen Universalgrammatik nicht

1. Im Folgenden soll der Langue-Begriff übernommen werden, da die Beschränkung des Kompetenz-Begriffes auf den idealen Sprecher-Hörer inadäquat erscheint. 
erforderlich, wenn vergleichbare Lebensbedingungen und mental-strukturelle Gegebenheiten zu vergleichbaren Verhaltensweisen führen und damit ähnliche sprachliche Strukturen erklären könnten (Elsen 2000). Ausführliche Datensammlungen könnten hier wesentlich neue Erkenntnisse liefern. So liegen mittlerweile Untersuchungen vor, die zeigen, dass manche sogenannte Ausnahme varietätenbedingt häufiger auftritt, als bisher angenommen und dass distinktive Kategorien, wie von strukturalistischen und generativen Ansätzen postuliert, der Datenlange bei weitem nicht gerecht werden (u. a. Elsen 2004, 2008a, Eichinger et al. 2008).

\section{Zur Struktur von Wortbildungskategorien}

\subsection{Statische vs. dynamische Typologien}

Wenn statt schreibtischbasierter Überlegungen Datensammlungen im Vordergrund stehen, folgt aber:

(i) Warum müssen einzelne theoretische Ansätze überhaupt verteidigt werden, wenn sie nicht zu den Daten passen?

(ii) Daten können sich nicht je nach Theorie unterscheiden, wenn sie solide und zuverlässig erhoben bzw. elizitiert wurden.

(iii) Auch die Ansicht de Saussures, die Langue konstituiere sich auf der Basis der Parole, kann durchaus in Frage gestellt werden.

Offensichtlich ist, dass durch eine Beschränkung des Analyseschwerpunktes auf die Langue Ausnahmen und unprototypische Belege bzw. Regeln, die wichtige Hinweise auf Markiertheit, Kreativität und Expressivität geben, unberücksichtigt bleiben (vgl. Zwicky \& Pullum 1987, Baldi \& Dawar 2000). Aus dieser Vorgehensweise resultieren verschiedene Probleme: In der Regel legen Untersuchungen nur unzureichende variative Maßstäbe an, indem sie von schriftlichen Quellen und determinierten soziolinguistischen Variablen ausgehen, die als repräsentativ für die Langue erachtet werden. Daraus folgt, dass Kategorien für Wortbildungseinheiten und -modelle vielfach stark generalisierend für den (vermeintlichen) Standard postuliert werden. Das Varietätenspektrum des Deutschen wird somit nahezu vollständig ausgeblendet. Die Sprache ist ein heterogenes Gebilde, das sich aus unterschiedlichen Varietäten zusammensetzt. In vielen Fällen kristallisieren sich monolinguale Besonderheiten erst durch eine polylinguale Betrachtung heraus. Konkrete Daten aus verschiedenen Erhebungsbereichen sind unabdingbar, um hier Erkenntnislücken zu schließen.

Die klassische Vorstellung diskreter Kategorien, die durch eine Gruppe von Merkmalen charakterisiert sind, über die alle Mitglieder in 
gleicher Weise verfügen, kann längst nicht mehr als kognitiv realistisch gelten, denn viele Eigenschaften sind mehr oder weniger treffend bzw. gut. Vor allem Kriterienbündel dürfen statt lediglich isolierter Merkmale eine distinktive Relevanz entwickeln, (vgl. Elsen 2007, 2008b). Es existieren also zentrale (,gute“, „optimale“) und periphere (,schlechte") Beispiele einer Kategorie mit zahlreichen interpolaren Abstufungsgraden (vgl. Michel 2006 und i. Dr. a). Solche Abstufungen lassen sich hinreichend nur durch ko- und kontextuelle Faktoren explizieren.

In Bezug auf den zuletzt genannten Aspekt rechtfertigen ähnliche Belege zunächst eine eigene Kategorie, beispielsweise um die Systematizität, die hinter solchen „Ausnahmen“ steckt, besser fassen zu können. Jedoch entziehen sich diese Erscheinungen oft einer direkten Definition, weshalb die Annahme so genannter Sonderkategorien (z. B. Affixoide, Zusammenbildungen, Zusammenrückungen, Konfixe etc.) in der Forschung nicht unumstritten ist. Alternativen bestehen etwa in der Zuordnung der fraglichen Belege $\mathrm{zu}$ den vermeintlich „etablierten“2 Kategorien (vgl. Donalies 2007). Erweist sich einerseits eine solche Reduktion forschungspraktisch als durchaus sinnvoll und notwendig, birgt sie andererseits jedoch die Gefahr, dass interne Kategorienstrukturen nicht ersichtlich werden. Vielmehr suggeriert sie eine kategoriale Homogenität, die der empirischen Überprüfung bedarf.

\subsection{Prototypentheorie}

Sehr eindrücklich lässt sich diese Problematik gegenwärtig an der Kategorie Konfix illustrieren. Konfixe gelten allgemein als gebundene nichtnative Grundmorpheme, die basis- und kompositionsgliedfähig sind, über eine lexikalisch-begriffliche Bedeutung verfügen, wortartvariabel und produktiv sind sowie in anderen Sprachen meist frei vorkommen: astro-(Astronaut), -mat (Automat). Diese, den Kernbestand an deutschen Konfixen abdeckenden, Definitionseigenschaften trennen Konfixe von Suffixen einerseits und von Wörtern andererseits. Jüngst ins Blickfeld geratene Einheiten wie z. B. -minator (Ebayminator) oder -tainment (Weintainment), die sich nicht eindeutig in eine Wortbildungskategorie einordnen lassen, stellen eine Herausforderung für die systembezogene statische Definition dar (Michel 2007 und i. Dr. a). Die traditionellen, auf die Langue bezogenen statischen und abstrakten Definitions- und Typologisierungsmuster erweisen sich als unzureichend. Das gilt auch für die gar nicht vorhandene klare Grenzlinie zwischen Morphologie und

2. Was genau unter „etabliert“ zu verstehen ist, bleibt in der Regel ungeklärt. Handelt es sich hierbei um die Kategoriengröße, die Akzeptanz, die Bekanntheit etc.? Wo beginnt Etabliertheit und wo hört sie auf? Dies müsste empirisch geklärt werden. 
Phonologie, denn hier kommt es zu systematischen Übergangsbereichen (Elsen 2008a). Schon die Prager Schule arbeitete mit den Begriffen Zentrum und Peripherie und stellte die grundsätzliche Gleichrangigkeit von Mitgliedern einer Kategorie in Frage. Adäquater scheint daher eine Typologisierung mit Hilfe der Prototypentheorie zu sein, die sich als empirische Meta-Theorie konsequent am Sprachgebrauch, also der Parole, orientiert (vgl. u. a. Mangasser-Wahl 2000). Eine Möglichkeit der Ermittlung prototypischer und unprototypischer Charakteristika stellt der Frequenzansatz dar. Mittels umfangreicher und hinsichtlich soziologischer Variablen exakt definierter gesprochener und geschriebener Korpora lassen sich Prototypenanordnungen für bestimmte Wirklichkeitsausschnitte erstellen. Gerade im metasprachlichen Bereich sind Verfahren der Introspektion und der Rezipientenbefragung nur bedingt tauglich. Dennoch scheint der Status der Frequenz bislang nicht hinreichend geklärt. Weitere Faktoren neben der Typen- und der Tokenfrequenz bei der Bestimmung von „zentral“ und ,peripher“ dürften etwa die Länge eines Ausdrucks oder die Ikonizität sein.

In einem weiteren Schritt können die Ergebnisse funktional interpretiert werden. Als primär kognitive Domäne lassen Prototypologien Rückschlüsse auf kognitive Strukturen und Prozesse zu, so dass die Unterschiede zwischen prototypisch und unprototypisch zunächst einmal unter Rekurs auf die Kognition zu beantworten wären. Morphologische Strukturen und Prozesse sind demnach ein Abbild entsprechender kognitiver Strukturen und Prozesse (Morphokognition). Neben kognitiven spielen auch sozio-pragmatische Faktoren eine nicht unwesentliche Rolle, wenn es um die Erklärung von Markiertheitsverhältnissen geht (Morphosoziopragmatik) (vgl. Michel i. Dr. b). Damit kann die Prototypentheorie als eine Möglichkeit aufgefasst werden, die Spannbreite der Daten zu ordnen und zu verstehen. Sie lässt sich nicht nur mit den aktuellen kognitiven Ansätzen in Einklang bringen, die davon ausgehen, dass die Sprachfähigkeit auf allgemeine kognitive und perzeptuelle Fähigkeiten zurückzuführen und zumindest teilweise durch sie motiviert ist - damit erübrigt sich zudem die aus methodischer Sicht mächtige Annahme einer UG. Vielmehr lassen sich durch die konsequente und systematische Berücksichtigung von Sprachgebrauchsdisziplinen wie etwa Korpuslinguistik, Gesprächs- und Textlinguistik, kognitive Linguistik sowie Soziolinguistik und Pragmatik Typologien mithilfe der Prototypentheorie als sprachrealitätsnah und dynamisch beschreiben (vgl. Elsen/Michel 2007).

\section{Schluss}

Die Wortbildungsforschung hat sich traditionell mit systemlinguistischen Fragestellungen befasst und Aspekte des Sprachgebrauchs lediglich ge- 
streift. Dadurch entstehen methodologische sowie theoretische Herausforderungen für die Wortbildung einerseits und für Disziplinen des Sprachgebrauchs andererseits. Wenn ,unterschiedliche Datenausschnitte unterschiedliche Theorien liefern" (ZS-Redaktion), dann sind die Theorien inadäquat - und die Zukunft hat den Daten adäquate Beschreibungen und Erklärungsansätze gegenüber zu stellen.

\section{Literatur}

Baldi, Philip \& Chantal Dawar (2000). Creative processes. In Morphology, an international handbook on inflection and derivation, Volume 1, Gert Booij, Christian Lehmann \& Joachim Mugdad (eds.), 963-972. Berlin, New York: de Gruyter.

Chomsky, Noam (1969). Aspekte der Syntax-Theorie. Frankfurt am Main: Suhrkamp Verlag.

Donalies, Elke (2007). Basiswissen Deutsche Wortbildung. Tübingen - Basel: Francke.

Dressler, Wolfgang U. \& Lavinia Merlini Barbaresi (1997). Morphopragmatics. In Handbook of Pragmatics, Jef Verschueren, Jan-Ola Östman, Jan Blommaert \& Chris Bulcaen (eds.), 1-14. Amsterdam, Philadelphia: Benjamins.

Eichinger, Ludwig M., Meike Meliss, María J. D. Vázquez (2008). Wortbildung heute. Tendenzen und Kontraste in der deutschen Gegenwartssprache. Tübingen: Narr.

Elsen, Hilke (1999). Ansätze zu einer funktionalistisch-kognitiven Grammatik. Konsequenzen aus Regularitäten des Erstspracherwerbs. Tübingen: Niemeyer.

Elsen, Hilke (2000). The structure of meaning. Onomasiology Online 1 [http://www. onomasiology.de] s. v. Elsen1-00/1.

Elsen, Hilke (2004). Neologismen. Formen und Funktionen neuer Wörter in verschiedenen Varietäten des Deutschen. Tübingen: Narr.

Elsen, Hilke (2005). Deutsche Konfixe. Deutsche Sprache 33: 133-140.

Elsen, Hilke (2008a). Phantastische Namen. Die Namen in Science Fiction und Fantasy zwischen Arbitrarität und Wortbildung. Tübingen: Narr.

Elsen, Hilke (2008b). Kontaminationen im Randbereich der deutschen Grammatik. Deutsche Sprache 2008 (2): 1-13.

Elsen, Hilke \& Sascha Michel (2007). Wortbildung im Sprachgebrauch. Desiderate und Perspektiven einer etablierten Forschungsrichtung. Muttersprache 2007 (1): 1-16.

Fleischer, Wolfgang \& Irmhild Barz (1995). Wortbildung der deutschen Gegenwartssprache. Unter Mitarbeit von Marianne Schröder. 2. Auflage. Tübingen: Niemeyer.

Köpcke, Klaus-Michael (1993). Schemata bei der Pluralbildung im Deutschen. Versuch einer kognitiven Morphologie. Tübingen: Narr.

Krahe, Hans \& Wolfgang Meid (1967). Germanische Sprachwissenschaft III. Wortbildungslehre. Berlin: Walter de Gruyter.

Levinson, Stephen C. (1994). Pragmatik. 2., unveränderte Auflage. Tübingen: Niemeyer.

Löffler, Heinrich (2005). Germanistische Soziolinguistik. 3., überarbeitete Auflage. Berlin: Schmidt.

Mangasser-Wahl, Martina (Hg.). (2000). Prototypentheorie in der Linguistik. Anwendungsbeispiele - Methodenreflexion - Perspektiven. Tübingen: Stauffenburg.

Michel, Sascha (2006). Kurzwortgebrauch. Plädoyer für eine pragmatische Definition und Prototypologie von Kurzwörtern. Germanistische Mitteilungen 64: 69-83.

Michel, Sascha (2006). Vom Terminator zum TORminator. Die Wortbildungseinheit -minator: Strukturelle und sozio-pragmatische Analysen. Muttersprache 2006 (4): 289-307.

Michel, Sascha (im Druck a). Das Konfix zwischen ,Langue' und ,Parole'. - Ansätze zu einer sprachgebrauchsbezogenen Definition und Typologie. In Studien zur Fremdwortbildung, Peter O. Müller (Hg.). Hildesheim et al.: Olms. 
Michel, Sascha (im Druck b). Randphänomene der Wortbildung im Deutschen. Diachrone und synchrone Untersuchungen. Stuttgart: ibidem (= PGL 2).

Saussure, Ferdinand de (1967). Grundfragen der Allgemeinen Sprachwissenschaft. 2. Auflage. Charles Bally \& Albert Sechehaye (Hgg.). Berlin, New York: de Gruyter.

Schmid, Hans-Jörg (2000). Methodik der Prototypentheorie. In Prototypentheorie in der Linguistik. Anwendungsbeispiele - Methodenreflexion - Perspektiven, Martina Mangasser-Wahl (Hg.), 33-53. Tübingen: Stauffenburg.

Zwicky, Arnold M. \& Geoffrey K. Pullum (1987). Plain morphology and expressive morphology. In Proceedings of the Thirteenth Annual Meeting of the Berkeley Linguistic Society, John Aske, Natasha Beery, Laura Michaelis \& Hana Filip (eds.), 330-340. Berkeley: Berkeley Linguistic Society.

Hilkee@lrz.uni-muenchen.de Universität München michel@uni-koblenz.de Universität Koblenz-Landau 